\title{
Computational and Behavioral Trust Assurance by Utilizing Profile-based Risk Assessments: The CATM Methodology
}

\author{
Khalil Abuosba \\ Ted Rogers School of Information Technology Management \\ Ryerson University
}

\begin{abstract}
Communication within a distributed system may be abstracted as an interaction of two endpoints of communications that traverse through intermediary nodes. With the explosion of new applications and services in the Internet as well as the new capabilities of the sensor-based and data-driven services, that are described as the Internet of Things (IoT), a major requirement arises that should facilitate trust between endpoints of communication. Security issues arise due to occurrence of incidents that compromise computational and behavioral trusts. In distributed systems endpoints of communications might consume or provide services as well as exchange messages between senders and recipients. A major issue in all types of interactions is to convey trust between any two points of communication. These systems are deployed based on different architectures. Within the Internet systems require assurance prior communications processes occur. This research introduce a trust management approach that can be utilized by any node that communication within a distributed system. The methodology utilizes a profile-based approach to achieve high level of assurance process that can achieve any security requirement including confidentiality, availability, authenticity, integrity, and non-repudiation. It allows the abstraction and inclusion of different attributes of both computational and behavioral trusts. The approach is extensible in nature, where modular security requirements are added as needed. The methodology can be utilized as a gatekeeper and as an access control mechanism. The methodology is an application layer solution of the OSI model that defines five building blocks: profile definition, profile abstraction, profile exchange, profile verification, and trust evaluation. The methodology requires extensible implementation in order to guarantee interoperability.
\end{abstract}

\section{Introduction}

"Trust Management" is defined as a unified approach utilized for the purpose of specifying and interpreting security policies, credentials, and relationships which allow direct authorization of security-critical actions [1]. In Macmillan Dictionary, trust is defined as to have faith or confidence in the integrity, honesty, reliability. Operational trust which is defined as reliance. Trust may be classified as either internal or operational [3]. Figure 1 maps security requirements attributes to distributed systems process criteria, Achieving trust between endpoints of communication depends on successful completion of risk assessment of the computing environment's endpoints of communications.

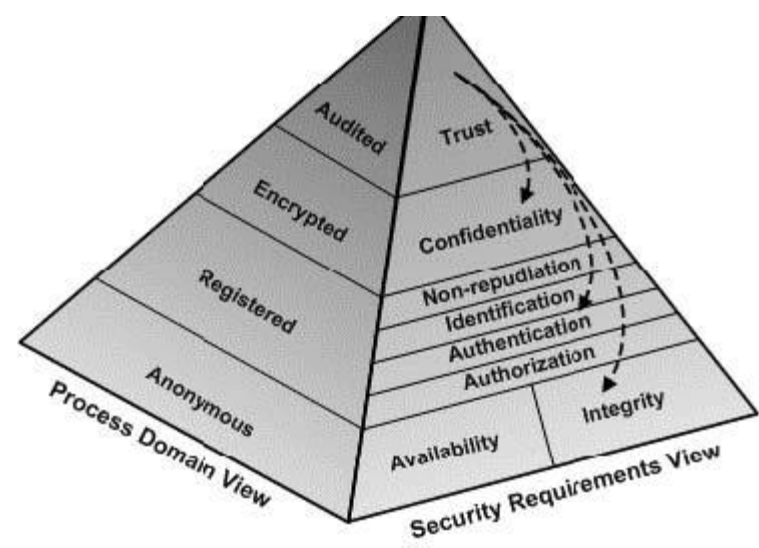

Figure 1. A conceptual model that depicts mapping of security requirements attributes to distributed processes criteria

\section{The Impact of Optimum Trust in Distributed Systems Security and IoT Services Delivery}

Operational trust management approaches are classified as Policy-based Trust Management [4], Reputation-based Trust Management [5], Capabilitybased Trust Management [5], Certificate-based Trust Management [6], and Profile-based Trust Management. In this research I have introduced Profile-based Trust Management that targets trust management between endpoints of communication and relies on risk assessment as a major parameter of its deployment.

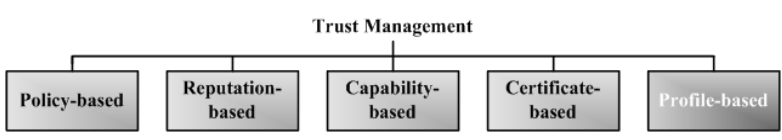

Figure 2. Types of Trust Management

Additionally, hybrid type of trust would be a combination of one or more of theses types of trusts. This hybrid type of trust may be abstracted as a combination of behavioral trust and computational 
trust. Behavioral trust defines trust relations among people and organizations; computational trust, among devices, computers, and networks. Behavioral trust defines trust relations among people and organizations; computational trust, among devices, computers, and networks [7], Glingor and Wing argue new trust relations increase the pool of usable services, remove cooperation barriers among users, and at the very least, take advantage of "network effects."; they also argue that network infrastructures that support the establishment of behavioral trust, which lower risk and betrayal aversion between untrusting parties and increase beliefs in trustworthiness between these parties, will spur establishment of unmediated trust relations, and as a consequence create new economic value. [8]. In the new eras of cloud computing and the emergent applications of the Internet of Things (IoT), new types of trust are required to address the attributes of behavioral and computational trusts. In this research we see an opportunity to introduce a layered trust model that assert overall trust that we call optimum trust based on the behavioral trust (unmediated) and computational trust (mediated). Optimum trust allows variability based on predefined attributes and operations.

\section{Data-Driven Profile-based Trust Assurance}

Profile-based trust management is viewed as an approach that addresses computing environment configuration compliance based on the process of defining a computing profile of identified attributes abstracted from the environment resources lists and logs. This method is used in order to resolve the operational communication authorization trust problem in which vulnerabilities reduction is achieved implicitly; reducing the system surface of operation reduces the exposure to some possible vulnerabilities. Reduction of the attack surface may be achieved by [8] reducing the number of system actions, remove a known or potential system vulnerability by strengthening the pre- and postconditions of a system action, eliminate an entire attack class, and reduce the number of instances of an attack class. Profile-based trust management approach is based on generating authorization credentials (and possibly authentication) based on the host environment variables profile in which collaboration occurs for the purpose of fulfilling operational trust between communications endpoints. Profile-based trust management has the advantage of reducing the operational risk based on the size of the active preconfigured resources. It is considered to be an enforcement utility that shall provide an assurance vehicle to operational trust. What distinguishes this approach from others? Rather than building the trust credentials based on authentication elements such as public key infrastructure, this approach builds trust definition based on the profile attributes; these attributes are abstracted from the endpoint environment variables.

Operational trust require a party to make a rational decision based on knowledge of possible rewards for trusting and not trusting. Trust enables higher gains while distrust avoids potential loss. Therefore risk aversion is a critical parameter in defining trust"

\subsection{Operational Trust Credentials and Assertion}

Operational trust credentials are characterized to be abstracted elements based on operational environment elements rather than on identity verifications elements. Access to services or resources is granted based on compliance and successful verification. Evaluation of operational trust based on credentials may be performed by using one of two methods [4]:

1. The first is implementing a trust model that allow them to reason about the reliability of the service provider or service consumer.

2. The second is using agents (service consumer or service provider are represented as agents) that calculate the amount of trust they can place in their interaction partner. These agents must gather knowledge about their counterparts to be achieved by three methods:

- trust is computed as a rating of the level of performance of the trustee, a pre-agreed contract between a service user and provider.

- computed indirectly from recommendations provided by others.

- Socio-Cognitive trust which includes capability to determine motivations of other agents based on forming coherent beliefs.

\subsection{Operational Trust Enforcement}

Operational computing environment is viewed as a finite state machine that may be specified based on the operations requirements or goals. Reduction of exposure within an Operational Computing Environment leads to reduction of operational risk, hence more reliable and survivable computing environments are achieved. Operational security calls for conditioning events security [9] (represented as a system attribute) rather than marginal security (averaging and addressing different environments that might be explored by an intruder). I project the mentioned properties into our approach for defining an operational configuration profile based on restricting and diffusing capabilities. Operational 
trust mechanisms shall enforce characteristics of these environments based on operational profile configuration compliance. In Intrusion Detection Systems (IDSs) capabilities are defined as the basic units that describe the access that an attacker has obtained during an intrusion and capabilities are modeled based on a "6-tuple (source, destination, credential, action, service, property)" [10].

\subsection{Operational Configuration Profile}

Based on classifications of operational environments as threatening or non-threatening, I define an Operational Configuration Profile as a facility to be used for specifying environments boundaries. Strict and restricted boundaries are used to operate in non-threatening environments. Loose and un-restricted boundaries specify threatening environments. OCPs are specified based on abstraction of specified operational computing environment resources. Attributes of the OCP may be viewed as environment beliefs. Operational profile is characterized as an abstracted entity of the operational environment derived from the operational environment attributes memberships. The main goal of introducing Operational Configuration Profile is to specify environment boundaries and enforce compliance within an input space of a computing environment. The main goal of the research is to introduce a simple mechanism that may be deployed as a service within an operational environment possibly to be integrated in cloud computing interactions or in trusted computing bases environments rather than being built as a separate application. As a guideline for creating an OCP, I recommend the following:

1. Elements/attributes of the Operational Configuration Profile neither compromise the system itself nor cause exposure to system resources.

2. The OCP elements must not reveal information that may be used by intruders as capabilities.

3. Encrypted-OCPs may be used in secure environments where PKIs are deployed and where the OCP contains information that may be used by intruders as capabilities. However PKIs must not be a requirement for OCP implementations.

4. Implementation must be simple and do not consume computational resources.
5. For the purpose of lowering remote traffic overheads and addressing problems associated with intermediaries such man-inthe-middle attacks, OCP-Digests may be exchanged between endpoints rather than the entire OCP message.

6. Proof of compliance token is generated from the OCP by a hash function and may be combined with an identifier by using a combining function followed by an exchange of messages between endpoints leading to promotion of a more secure communication (suitable for applications where PKIs are not available).

7. Light weight implementation may be implemented based on inspecting the environment configurations based on system calls.

8. OCPs must be unified and extensible in order to guarantee interoperability.

9. OCPs must conform to separation of concern [11] paradigm in away where OCP is separated from the verification algorithm. Special computing concerns include Computational Algorithm Management and Optimization (i.e. distribution, realtime constraints, and persistence) or Special Requirements of Application Fulfillment (i.e. concurrency and location control). I classify concerns as basic concerns or special purpose concerns where a basic concern projects functional concerns that might be mapped to functional requirements, special purpose concerns may be grouped as aspects related to management and optimization of computational algorithms and a set of nonfunctional concerns that might be mapped to non-functional requirements. Benefits gained from deployment include high level of abstraction, better and ease of understanding of the functionalities, and it provides a facility for weak coupling.

10. The configuration profile may be generated and validated by using a state-detection algorithm; generation of such a profile is performed while the system is classified as a stable state/property (stable property example is a computation has terminated) [12]. Proof of compliance of a system state (system configuration) may be obtained by recording a system snapshot of the local systems variables defined within an Operational Configuration Profile. 
11. In order to achieve confusion/diffusion and compression [13], an ad-hoc hash function may be deployed. Ideally, we view Operational Configuration Profile as abstraction of a computing environment of a reflective system. Reflective systems are defined as ones, that are able "to inspect and to adapt its own internal behavior" [14]. I identify the OCP as a configuration profile of a reflective system in which re-configuring the environment assert modes of operation [15]. Reflective systems are characterized as being able to support architectural refinement including "the ability to modify network attributes using dynamic plug-ins"[15]. An example of such a system is the Genesis Kernel; these types of kernels are able to automate the creation, deployment and management of network architecture.

\section{Collaborative Anomalous Trust Management (Catm)}

Collaborative Anomalous Trust Management is built for the purpose of implicitly diffuse intrusion capabilities. CATM is based on anomaly detection by auditing a pre-defined operational profile of normal or ideal system behavior. I propose a solution to operational trust management and vulnerabilities management. Collaborative Anomalous Trust Management (CATM) is a methodology that may be utilized for the purpose of affirming trust between communications endpoints. In conjunction of a trusted computing base, zero knowledge protocol, a security policy enforcement mechanism, a layered trust credential validation process, and profile definition and encapsulation mechanism, CATM is defined. This methodology considers trust as a cumulative entity in which degrees of trust are asserted based on the process security goals. Ideally this methodology is suited for Service Oriented Architectures such as web services where service providers and consumers interact at different levels of security requirements. This methodology is optimized as risk management utility. Benefits include:

- $\quad$ Intrusion prevention and intrusion. capability diffusion.

- Vulnerabilities reduction.

- Operational trust assurance.

\subsection{CATM Modes of Operations}

I view Collaborative Anomalous Trust Management as a methodology that is deployed within a dynamic computing environment. Depending on the service requirements and application and in order to support flexibility and efficiency, CATM may be implemented in three modes: Loose, Strict, and Dedicated.

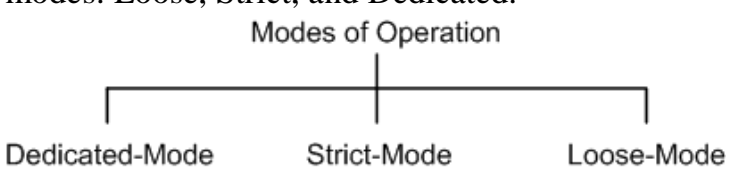

Figure 3. Types of Trust Management

Dedicated mode operates based on minimum functional requirements, strict mode operates based on user preference profile negotiated with the verifier, and loose mode operates based on verifying compliance of the trusted computing base where only embedded explicit bounded attributes are used and no profile elements are audited. Loose mode reflects that the communication channel is trusted and it can be deployed within any internal networks behind perimeter router.

\subsection{CATM Phases}

Collaborative Anomalous Trust Management isviewed as a methodology that is deployed within a dynamic computing environment. CATM comprises four phases:

1. Computing Base Definition Phase: Service and environment abstraction.

2. Generating Service Footprint Identifier Phase: Generating and exchanging a valid environment variable identifier (system footprint) using a hashing function.

3. Validating Service Footprint Identifier Phase.

4. Environment Verification and Control Phase: Interpreting the environment status based on the identifier.

\subsection{CATM Building Blocks}

CATM comprises five main building blocks: Trusted Computing Base, Zero Knowledge Protocol, Security Policy Enforcement Mechanism, Configuration Profile Definition and Encapsulation Mechanism, and Layered Trust Credentials Mechanisms:

1. The Trusted Computing Base (TCB) is the part of the system that is responsible for enforcing the information security policies of the system. All of the computer's hardware is included in the TCB, but a person administering the system should be concerned primarily with the software components of the TCB [16].

2. Zero knowledge protocol [17] which is built based on the notion that zero knowledge is transferred between endpoints of communication, this protocol may be eased 
little bit by deployment of a Minimum Disclosure Protocol.

3. Security policy enforcement mechanism which a trusted computing base has the ability to enforce correctly a unified security policy.

4. Configuration profile definition and encapsulation mechanism in which are built based on computing environment variables.

5. Layered trust credentials verification based on set of credentials specified on the system profile.

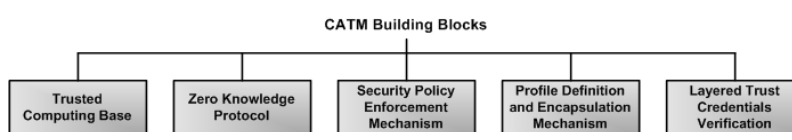

Figure 4. CATM Building Blocks

\section{The Asynchronous Environment Profile Methodology (ACEPUM)}

\section{Computing Unification}

Based on CATM, ACEPUM is introduced for the purpose of building configuration profile definitions. The applications of use include:

- $\quad$ A trust assurance and access control utility in $\mathrm{B} 2 \mathrm{~B}$ machine-to-machine interactions such as web services.

- ACEPUM is optimized for Peer-to-Peer (P2P) communication model which is characterized by dynamic symmetry; each party exposes a set of comparable functionality and any party can initiate a communication session at any time [18]; however, this trust management methodology can be used in any client/server architectural deployment.

- Implementations may utilize specific intelligent agent properties such as selfmanaging, self-modifying, proactive, reactive, autonomic, collaborative, and incorporates cross communications capabilities.

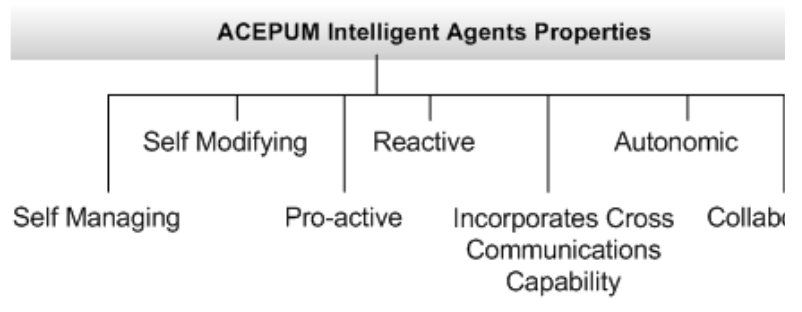

Figure 5. ACEPUM Intelligent Agents Properties

A collaboration diagram that depicts the process of service authorization in a strict mode deployment is shown in figure 5. Channel communications are blocked whenever trust is not needed hence services are not provided to the requester; this is usually determined by evaluating computational trust. Here, another concern arise, whenever behavioral trust is not needed due to suspicious of user behavioral, the system should also isolate the user or sender by blocking communication with that entity.

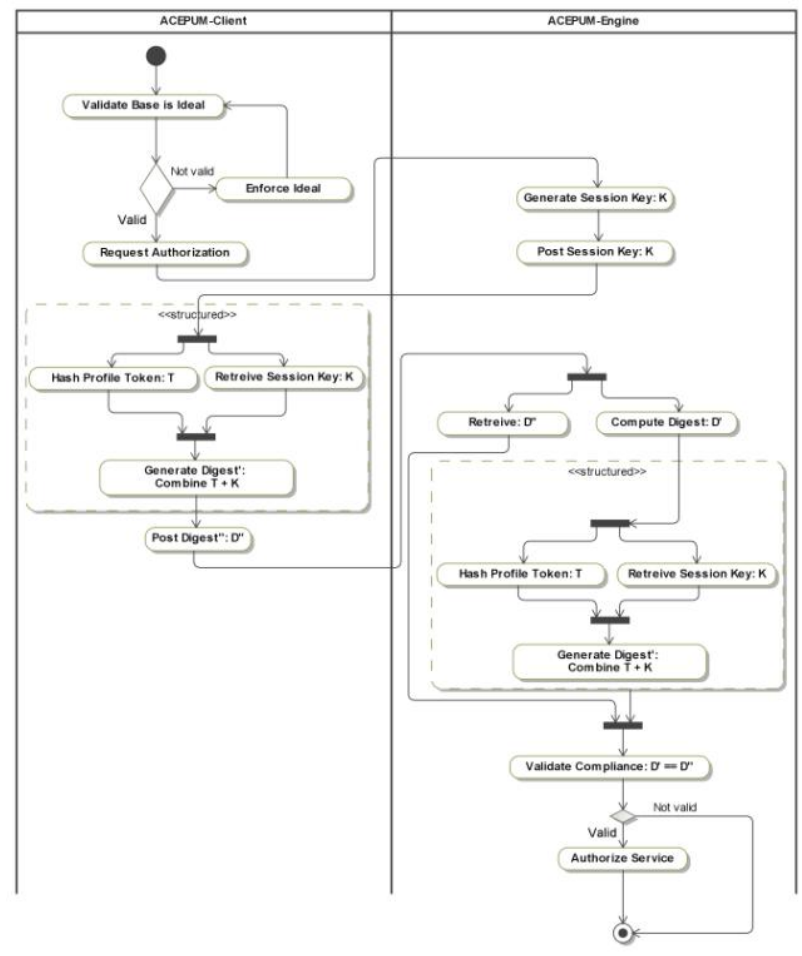

Figure 6. Collaboraction diagram of ACEPUM in Strict Mode

ACEPUM comprises the following advantages:

- It provides a low computational overhead using a message based solution.

- It utilizes SOA properties to achieve its goals of design [19].

- It provides ability to customize computational profiles.

- It does not compromise systems which are being audited as systems scanning is performed locally rather than being scanned remotely.

- Environment may be audited promptly at runtime using a snapshot protocol.

- No significant consumption of network bandwidth due to the only identifiers are exchanged between endpoints of communication.

ACEPUM system may abstracted using Data Flow Diagrams. Figure 5. Depicts how data flow while interacting with the main entities of the system. 


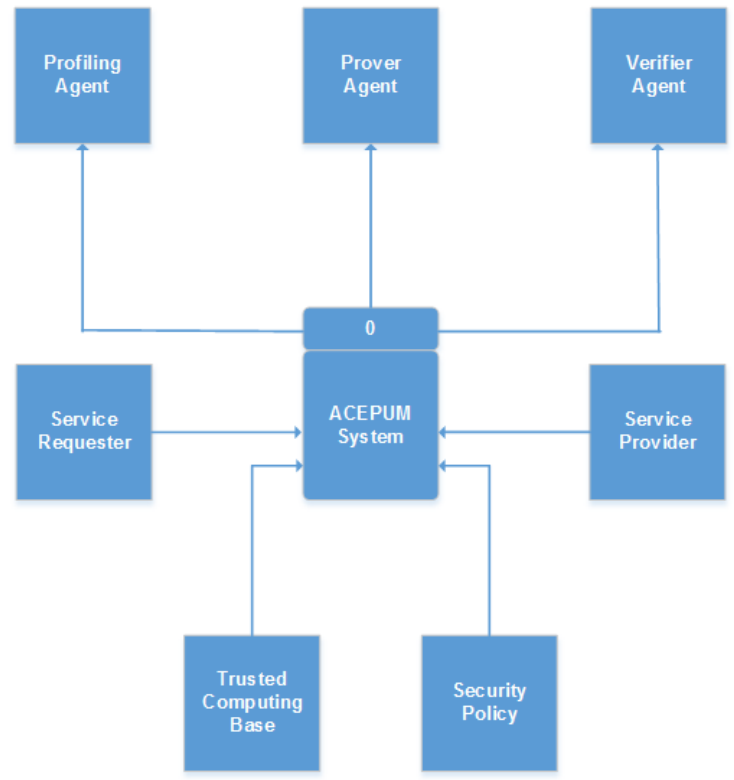

Figure 7. Context Data Flow Diagram of ACEPUM

\section{Achieving Nonmediated Behaviroal Trust and the Optimum Trust}

Nonmediated behavioral trust is usually compromised by the assumption that achieving computational trust would grant similar level to behavioral trust. The email trust model is an example of false expectations: the widespread user expectation that electronic mail would mirror the trust model of physical mail (e.g., authenticity, confidentiality, nonrepudiation of receipt, delivery in bounded time) has misled many unsuspecting users into accepting spam, misleading ads, and malware [7]. Behavioral trust might be comprised due to events and actions taken by a requester and the counter measure is to isolate the requester; however, determining suspicious activities requires monitoring and logging. Anomaly based screening of activities should be sufficient to determine penetrators. Clearances and classifications would one way to achieve deployment flexibility of non-anonymous processes; this can be accomplished by deployment of access control security policies such as Bell LaPadula [20] and the Biba models [21], [22]. The optimum trust is achieved by abstraction (possibly quantifying a level) joining the computational trust and behavioral trust into a single entity that is measured using a function that interprets trust based on threshold levels; these levels act as credentials that can be utilized for access control purposes. Achieving optimum trust requires five major processes:

- Profile definition (Computational and Behavioral).

- Profile Abstraction

- Profile Exchange.

- Profile Verification.
- $\quad$ Trust Evaluation.

\section{Conclusion and Future Work}

In this research we have introduced a profilebased trust management methodology. The methodology builds upon Service Oriented Architecture properties. It introduces a conceptual model that asserts trust between endpoints of communication. In future work, formal definition of the processes as well as the optimum trust will be defined, requirements decompositions including development of level-0 logical and physical diagrams are addressed. Formal sequence diagrams will also be developed. More work will be conducted that shall investigate the role of machine learning in the design of learning agents. A Formal model of the system will also be addressed.

\section{References}

[1] M. Blaze, J. Feigenbaum, and J. Lacy, "Decenteralized Trust Management" Proceedings of the IEEE Conference on Security and Privacy, pp. 164-173 May 1996).

[2] Fred B. Schneider, Enforceable Security Policies, ACM Transactions on Information Systems, February 3, 2003.

[3] D.E. Zand, "Trust and Managerial Problem Solving," Administrative Science Quarterly, vol. 17, 1972, pp. 229-239

[4] Vinicius da S. Almendra1, Daniel Schwabe, Realworld Trust Policies, Semantic Web and Policy Workshop 4th International Semantic Web Conference, November 2005, Galway, Ireland.

[5] Daniel Olmedilla, Omer F. Rana, Brian Matthews, and Wolfgang Nejdl. Security and trust issues in semantic grids. In Semantic Grid: The Convergence of Technologies, volume 05271 of Dagstuhl Seminar Proceedings. Internationales Begegnungs- und Forschungszentrum (IBFI), Schloss Dagstuhl, Germany, July 2005.

[6] The Orange Book, The US DoD Trusted Computer Systems Criteria, 1982, DoD2500.28-STD.

[7] V. Gligor and J.M. Wing, Towards a Theory of Trust in Networks of Humans and Computers, Security XIX, 19th International Workshop, Cambridge, UK, Springer, March 2011.

[8] P. Zimmerman, PGP User's Guide, MIT Press, Cambridge, 1994. 
[9] Lujo Bauer, Jarred Ligatti and David Walker, More Enforceable Security Policies, Department of Computer Science Princeton University Princeton, NJ 08544, Tech Report TR-649-02 June 17, 2002.

[10] J. Zhou, M. Heckman, B. Reynolds, A. Carlson, and M. Bishop, "Modeling Network Intrusion Detection Alerts for Correlation," ACM Transactions on Information and System Security 10(1) pp. 1-31 (Feb. 2007).

[11] Walter Hürsch, Cristina Lopes. Separation of concerns Northeastern, University technical report NU-CCS-95-03, 1995.

[12] K. Manay Chandy, and Leslie Lamoprt, Distributed Snapshots: Determining Global States of Distributed Systems, ACM Trans. on Computer System 1985.

[13] Vadim Lyubashevsky, Daniele Micciancio, Chris Peikert, Alon Rosen, Provably Secure FFT Hashing, NIST 2nd Cryptogaphic Hash Workshop, Santa Barbara, CA, USA, 2006.

[14] Jan de Meer, On The Construction of Reflective System Architectures GMD-FOKUS, Kaiserin-AugustaAllee 31, D-10589 Berlin.

[15] Andrew T. Campbell, and Michael E. Kounavis, Toward Reflective Network Architecture, COMET Group, Technical Report, Center for Telecommunications Research, Columbia University.

[16] Butler Lampson, Martin Abadi, Michael Burrows, Edward Wobber, ACM Transactions on Computer System, 1992.

[17] Blum, Manuel; Feldman, Paul; Micali, Silvio (1988). "Non-Interactive Zero-Knowledge and Its Applications". Proceedings of the twentieth annual ACM symposium on Theory of computing (STOC 1988): 103-112.

[18] S. A. Hofmayji, and S. Forrest, Intrusion Detection using Sequences of System Calls, Journal of Computer Security, Volume 6, 1998, Page 151-180.

[19] Abuosba K., El-Sheikh A., Formalizing Service Oriented Architectures, IEEE IT Professional Magazine, Volume 10, Issue 4, July-Aug. 2008 issue, pages: 34-38, August 2008.

[20] D. E. Bell and L. J. La Padula. Secure computer system: Unified exposition and Multics interpretation. Technical Report ESD-TR-75-306, Mitre Corporation, Bedford, MA, March 1976.

[21] Biba, K. J. "Integrity Considerations for Secure Computer Systems", MTR-3153, The Mitre Corporation, April 1977.

[22] RFC 1457, Security Label Framework for the Internet, May 1993. 